\section{Utilização de medicamentos antes e durante a gestação: prevalência e fatores associados}

\author{
Use of medicines before and during pregnancy: \\ prevalence and associated factors
}

\author{
Utilización de medicamentos antes y durante la \\ gestación: prevalencia y factores asociados
}

Débora Bomfim Costa 1

Helena Lutescia Luna Coelho 1

Djanilson Barbosa dos Santos 1,2

doi: 10.1590/0102-311X00126215

\section{Resumo}

Estudo transversal aninhado à coorte prospectiva com o objetivo de avaliar a prevalência e os fatores associados à utilização de medicamentos em gestantes antes e durante a gravidez em município do interior da Bahia, Brasil. As informações foram coletadas mediante um questionário estruturado aplicado às gestantes no momento do acompanhamento pré-natal em unidades de saúde do município. A prevalência para consumo de medicamentos antes e durante a gestação foi 52,1\% e 84,7\%, respectivamente. Após análise, os seguintes fatores estavam associados à utilização de medicamentos antes da gestação: $\geq$ 30 anos de idade, as não pretas, as que iniciaram o pré-natal depois do 10 trimestre e as que fazem parte da classe econômica C/D/E. Há um aumento de prevalência de utilização de medicamentos durante a gestação entre as gestantes com escolaridade $\geq 11$ anos de estudo, ter feito mais de três consultas pré-natais e ter algum problema de saúde. As gestantes estão expostas ao uso de medicamentos antes e durante a gestação apesar da carência de informações seguras que fundamentem o uso de medicamentos nessa fase, e esse uso está associado a fatores relativos ao acompanhamento pré-natal, sugerindo-se a inclusão mais ativa do farmacêutico na equipe para orientação e apoio ao uso racional de medicamentos.

Uso de Medicamentos; Gravidez; Farmacoepidemiologia

\section{Correspondência}

D. B. Costa

Universidade Federal do Ceará.

Rua Capitão Francisco Pedro 1210, Fortaleza, CE 60431-327,

Brasil.

bomfim.debora@gmail.com

1 Universidade Federal do Ceará, Fortaleza, Brasil. 2 Centro de Ciências da Saúde, Universidade Federal do Recôncavo da Bahia, Santo Antônio de Jesus, Brasil. 


\section{Introdução}

A utilização de medicamentos por gestantes passou a ser pauta de uma grande discussão a partir da década de 1950, com a tragédia da talidomida, um medicamento sedativo utilizado no tratamento de náuseas e vômitos durante a gravidez, que levou ao nascimento de cerca de 10 mil crianças com focomelia, uma má-formação congênita, que até aquele momento era considerada rara 1 .

Antes desse episódio, acreditava-se que a placenta era uma barreira que protegia o feto de qualquer ação farmacológica; hoje, porém, sabe-se que a maioria dos fármacos utilizados por gestantes atravessam a placenta e atingem a corrente sanguínea do feto 2,3 , que, não possuindo os sistemas corporais plenamente desenvolvidos, está mais sujeito a efeitos negativos não esperados 4 .

A não inclusão de mulheres em idade fértil nos ensaios clínicos de novos fármacos, bem como as evidências de que os estudos de toxicidade em animais não preveem com segurança os efeitos em humanos, justificam os cuidados que se deve ter com o uso de medicamentos em gestantes 5 . No entanto, dado que nas sociedades modernas o uso de medicamentos tornou-se prática costumeira e de dimensão crescente, a exposição de conceptos em períodos iniciais da gestação é um evento de possibilidade frequente, especialmente em gravidezes não planejadas 6 . Tais fatores ressaltam a importância da realização de estudos observacionais sobre o uso de medicamentos na gravidez, que têm se intensificado nas últimas décadas 6 .

Conforme esses estudos, as prevalências de uso de medicamentos antes da gestação em países europeus variam de 29,9\% a 43\%, sendo os grupos terapêuticos mais utilizados os antibacterianos de uso sistêmico, hormônios sexuais e anti-inflamatórios não esteroides 6,7,8,9,10. Investigação realizada em cidade do Sul do Brasil mostrou que 46,7\% das mulheres estavam em uso de medicamentos no momento do diagnóstico da gravidez. O percentual mais elevado de automedicação ocorreu antes do início do pré-natal $(64,9 \%) 11$.

Poucos trabalhos investigaram a associação entre os medicamentos utilizados antes da gestação e características socioeconômicas da mulher. Foi encontrado apenas um estudo sérvio em que idade materna ( $\geq 30$ anos), renda familiar per capita (> 300 Euros) e parição (mais de 1 filho) estiveram entre os fatores associados 12 .

Quanto ao uso de medicamentos durante a gestação, em países como França, Canadá, Estados Unidos e Brasil, foi verificado que mais de $80 \%$ das mulheres utilizam algum tipo de medicamento nesse período 13,14,15,16, entretanto se observa uma grande variação no perfil do uso entre os países, bem como nas características maternas associadas a esse uso 15,16,17,18,19. Alguns medicamentos recomendados para prevenção de problemas associados à gestação, incluindo ácido fólico, sulfato ferroso e vitaminas, certamente contribuem para essa alta prevalência 15.

No que se refere às associações de características maternas com o uso de medicamentos durante a gravidez, os resultados são ainda inconsistentes, enquanto alguns estudos apontam para o efeito positivo de número maior de anos de estudo, menor quantidade de filhos 17, ser solteira, parceiro com escolaridade básica 20; outros divergem desses resultados 17,18 .

Em razão do número limitado de achados sobre o uso de medicamentos antes e durante a gestação no Brasil, assim como inconsistências em relação aos fatores associados, o objetivo do presente trabalho foi avaliar a prevalência e fatores associados à utilização de medicamentos antes e durante a gravidez em gestantes atendidas no Sistema Único de Saúde (SUS), na cidade de Santo Antônio de Jesus, na Bahia.

\section{Metodologia}

Este estudo utilizou dados da pesquisa Fatores Maternos de Risco para o Baixo Peso ao Nascer, Prematuridade e Retardo do Crescimento Intrauterino, no Recôncavo da Bahia, realizada pelo Núcleo de Investigação em Saúde Materno-Infantil (NISAMI) do Centro de Ciências da Saúde, Universidade Federal do Recôncavo da Bahia, Brasil. Trata-se de um estudo transversal aninhado à coorte prospectiva com gestantes que realizaram o pré-natal das unidades básicas de saúde do SUS do Município de Santo Antônio de Jesus, no período de junho de 2012 a fevereiro de 2014. 
Santo Antônio de Jesus é uma cidade localizada no recôncavo baiano, a $187 \mathrm{~km}$ da capital Salvador. De acordo com o Censo Demográfico realizado em 2010 (http://www.ibge.gov.br, acessado em Jun/2015), a cidade possuía 90.985 habitantes, cerca de 48.020 do sexo feminino. A população estimada para 2014 foi de 100.550 habitantes. Do ponto de vista econômico, as principais atividades são agricultura, pecuária e comércio. Em 2011, o Produto Interno Bruto (PIB) per capita a preços correntes correspondia a R \$11.872,96. A prestação de serviços de saúde ocorre em 26 unidades de atendimento básico, 38 clínicas/centros de especialidade, cinco hospitais, duas policlínicas e ainda aloca a 4a Diretoria Regional de Saúde.

A pesquisa foi desenvolvida em todas as unidades básicas da zona urbana. As unidades básicas da zona rural foram excluídas por causa das dificuldades de acesso, provenientes da distância. Dessa forma, participaram deste estudo todas as mulheres gestantes com idade igual ou maior que 18 anos, residentes e domiciliadas na zona urbana do município, com qualquer idade gestacional, cadastradas no Sistema de Acompanhamento do Programa de Humanização no Pré-Natal e Nascimento (SISPRENATAL) e que realizaram ao menos um pré-natal.

O tamanho da amostra foi calculado para fornecer estimativas de prevalências de algumas características de interesse, o erro máximo aceitável foram 4 pontos percentuais, adotando-se o nível de 95\% de confiança. Para garantir que isso ocorra, foi dimensionada a amostra, considerando que a prevalência do uso de medicamentos é da ordem de 50\%, pois essa é a estimativa mais segura por corresponder ao maior tamanho de amostra que pode ser calculado. A amostra mínima necessária para garantir significância estatística foi de 600 gestantes, tendo em vista o poder do teste igual a $80 \%$. Como outras associações em estudo foram investigadas, adotou-se o maior número de gestantes para encontrar associação com a variável consultas de pré-natal, o que totalizou 891 mulheres, já adicionando $10 \%$ a mais de gestantes a fim de compensar não respostas e perdas, mas a amostra total foi de 1.091 mulheres.

As variáveis dependentes sobre a utilização de medicamentos antes e durante a gravidez foram medidas da seguinte forma: "A senhora estava usando algum remédio, antes de saber que estava grávida, para...: pressão alta, diabetes (açúcar alto no sangue), dor ou cólica, enjoo ou vômito, tosse, corrimento, infecção, problemas respiratórios e outros motivos?" e a questão "A senhora usou algum remédio, nesta gravidez, para...: pressão alta, diabetes (açúcar alto no sangue), dor ou cólica, enjoo ou vômito, tosse, corrimento, infecção, problemas respiratórios e outros motivos?", definindo o consumo de medicamentos durante a gestação. Os medicamentos foram codificados até o quinto nível conforme a Anatomical Therapeutic Chemical Classification System (ATC), da Organização Mundial da Saúde (OMS) 21.

As variáveis de exposição definem os fatores maternos de risco para o uso de medicamentos. Foram avaliadas as variáveis socioeconômicas, demográficas, maternas e de utilização de serviços de saúde sendo elas: idade materna (18-24 anos, 25-29, 30-45 anos), escolaridade materna ( $\leq 8$ anos de estudo, 9 a 11 anos, $>11$ anos), cor da pele (não pretas, pretas), situação conjugal (com companheiro, sem companheiro), número de gestações anteriores $(\geq 2,<2)$, início do pré-natal (durante o 1 o trimestre, após o 1 o trimestre), consultas pré-natal $(\leq 3,>3)$, renda familiar ( $>1$ salário mínimo, $\leq 1$ salário mínimo), classe econômica (A/B, C/D/E), história de aborto (sim, não), fumante (sim, não), ter algum problema de saúde (sim, não) sendo essa variável obtida pelo agrupamento das variáveis autorreferidas, ter ou não as seguintes patologias: anemia, asma, tuberculose, pneumonia, diabetes, hipertensão, doença renal, infecção urinária e hemorragias.

Todos os questionários preenchidos foram revisados pela equipe de supervisores, foi adotada uma validação dos dados por comparação das informações obtidas pelos questionários com aquelas registradas nos cartões de pré-natal. O entrevistador, sempre que necessário, regressou ao domicílio visitado para corrigir imperfeições da entrevista realizada. Vinte por cento das gestantes entrevistadas foram revisitadas pelos supervisores de campo, que reaplicaram parcialmente a entrevista. Os dados foram comparados com as entrevistas originais para avaliar sua qualidade, objetivando identificar eventuais imprecisões, erros sistemáticos ou mesmo fraudes. Foram consideradas perdas questionários com imprecisões que não puderam ser corrigidas.

A entrada dos dados foi feita em uma tela de entrada criada no programa Epidata, versão 3.0 (Epidata Association, Odense, Dinamarca), com sistema de checagem automática de consistência e amplitude. Após a revisão dos questionários, as informações foram compiladas em banco de dados informatizado para posterior análise estatística no programa Stata, versão 12.0 (StataCorp LP, College 
Station, Estados Unidos). Em seguida, o banco de informações foi editado para aferição da qualidade do processo de entrada de dados e correção dos erros detectados. Isso foi feito com o exame da distribuição de frequência de cada variável para identificação de valores fora de limites, checagem de valores inválidos, identificação de entrada em duplicata e checagem de dados incompatíveis ou contraditórios.

$\mathrm{Na}$ análise dos dados, foram estimadas as respectivas prevalências e frequências de utilização de medicamentos antes e durante a gestação, utilizando como denominador o total de gestantes e o total de medicamentos, respectivamente, segundo características demográficas, socioeconômicas e de saúde. Foi realizada uma análise bivariada para investigar a associação entre as variáveis independentes e a utilização de medicamentos antes e durante a gestação; a medida do desfecho foi expressa pela razão de prevalência (RP) com intervalo de 95\% de confiança (IC95\%). Na modelagem estatística, selecionaram-se as variáveis com valores de $\mathrm{p} \leq 0,20$ na análise bruta. A seguir, tais variáveis foram introduzidas no modelo de regressão de Poisson por meio do procedimento stepwise. Foram mantidas no modelo as variáveis que permaneceram significantes, aquela com valor $\mathrm{p} \leq 0,05$, depois do ajuste pelas outras variáveis. Foram ajustados modelos multivariados que permitiram calcular a RP ajustada (RPa) estimada por regressão de Poisson com variância robusta, com IC95\% 22.

A pesquisa Fatores Maternos de Risco para o Baixo Peso ao Nascer, Prematuridade e Retardo do Crescimento Intrauterino, no Recôncavo da Bahia foi submetido e aprovado pela Comissão de Ética e Pesquisa da Faculdade Adventista de Fisioterapia da Bahia (FAFIS), sob o protocolo no 4369.0.000.070-10. As gestantes foram orientadas quanto aos propósitos da pesquisa e sua metodologia e informadas sobre a confidencialidade dos dados pelo Termo de Consentimento Livre e Esclarecido (TCLE) que continha informações explícitas sobre a natureza e os objetivos do estudo. As entrevistas foram realizadas somente após assinatura do TCLE.

\section{Resultados}

Foram entrevistadas 1.099 gestantes, contudo, após revisão dos questionários, participaram da análise 1.091 gestantes, a idade das gestantes variou entre 18 e 45 anos, com média de 25,5 anos (DP $\pm 6,21)$ e mediana de 25 anos. Aproximadamente 50\% das gestantes têm menos de nove anos de estudo, 84,7\% são pretas e 23,5\% ganham menos de um salário mínimo. Metade das mulheres desse estudo não teve nenhuma gestação anterior, porém 61,1\% relataram não ter planejado a gravidez (dados não apresentados em tabela) e 24,5\% das gestantes têm história de aborto. Das gestantes, $83 \%$ iniciaram o pré-natal no 1 o trimestre com uma média de 3,30 \pm 3,02 consultas (Tabela 1), no momento da entrevista, 44\% das participantes deste estudo encontravam-se entre a 13a e a 24a semana de gestação (dados não apresentados em tabela).

Pouco mais da metade das gestantes $(52,1 \%, \mathrm{n}=567)$ utilizou medicamento antes da gestação, com um total de 873 medicamentos utilizados. Desses, $45 \%$ eram com prescrição médica e da enfermagem e 53,9\% foram relatos de automedicação, mas apenas 6,1\% das gestantes ainda estavam utilizando o medicamento no momento da entrevista (dados não apresentados em tabela). Antes da confirmação da gestação, as classes de medicamentos mais utilizados foram os analgésicos $(17,1 \%)$, hormônios sexuais (12,3\%), relaxantes musculares $(9,3 \%)$ e os anti-inflamatórios não esteroides $(5,8 \%)$ (Tabela 2$)$.

Em relação ao uso de medicamentos, 84,7\% $(\mathrm{n}=924)$ gestantes fizeram uso durante a gestação com um total de 2.229 medicamentos utilizados. Dentre eles, 56,6\% foram prescritos por médico e 34,7\% por enfermeiros. A automedicação foi referida por $13 \%(n=142)$ das gestantes (dados não apresentados em tabela). Durante o 1 o trimestre, $75,5 \%$ das gestantes começaram a utilizar algum medicamento, no 2 o trimestre essa prevalência caiu para 44,1\% e no 3 o trimestre o número foi de 6,4\% (dados não apresentados em tabela).

As gestantes utilizaram com maior prevalência durante a gravidez os medicamentos pertencentes às seguintes categorias ATC: sangue e órgãos hematopoiéticos $(71,4 \%)$, trato gastrintestinal e metabolismo (26,8\%), sistema nervoso central (21,9\%), sistema respiratório (9,3\%), sistema geniturinário e hormônios sexuais (4\%). No segundo nível de ATC, os mais frequentes utilizados pelas gestantes durante a gravidez foram os antianêmicos $(71,2 \%)$, analgésicos $(21,8 \%)$, medicamentos para distúrbios gastrointestinais $(19,5 \%)$ e anti-histamínicos sistêmicos $(8,9 \%)$ (Tabela 3$)$. 
Tabela 1

Características socioeconômicas e de saúde e prevalências da utilização de medicamentos antes e durante a gestação. Santo Antônio de Jesus, Bahia, Brasil, 2012-2014 ( $\mathrm{N}=1.091)$.

\begin{tabular}{|c|c|c|c|c|c|c|}
\hline \multirow[t]{2}{*}{$\begin{array}{l}\text { Características socioeconômicas e de } \\
\text { saúde }\end{array}$} & \multicolumn{2}{|c|}{ Gestantes } & \multicolumn{2}{|c|}{$\begin{array}{c}\text { Utilização de medicamentos } \\
\text { durante a gestação }\end{array}$} & \multicolumn{2}{|c|}{$\begin{array}{c}\text { Utilização de medicamentos } \\
\text { antes da gestação }\end{array}$} \\
\hline & n (\%) & IC95\% & n (\%) & IC95\% & n (\%) & IC95\% \\
\hline \multicolumn{7}{|l|}{ Idade materna (anos) } \\
\hline $18-24$ & $509(46,7)$ & $43,7-49,7$ & $427(83,7)$ & $80,2-86,8$ & $251(49,4)$ & $45,0-53,8$ \\
\hline $25-29$ & $290(26,6)$ & $24,0-29,3$ & $246(84,8)$ & $80,2-88,6$ & $151(52,3)$ & $46,3-58,1$ \\
\hline $30-45$ & $292(26,8)$ & $24,2-29,5$ & $251(86,0)$ & $81,4-89,7$ & $165(56,5)$ & $50,6-62,3$ \\
\hline \multicolumn{7}{|l|}{ Escolaridade (anos) } \\
\hline$\leq 8$ & $539(49,5)$ & $46,5-52,6$ & $443(82,2)$ & $78,7-85,3$ & $271(50,4)$ & $46,1-54,7$ \\
\hline $9-11$ & $487(44,8)$ & $41,8-47,8$ & $422(86,7)$ & $83,3-89,5$ & $256(52,6)$ & $48,0-57,1$ \\
\hline$>11$ & $62(5,7)$ & $4,4-7,2$ & $57(91,9)$ & $82,1-97,3$ & $38(62,3)$ & $49,0-74,4$ \\
\hline \multicolumn{7}{|l|}{ Cor de pele } \\
\hline Não pretas * & $162(15,3)$ & $13,2-17,6$ & $141(87,0)$ & $80,9-91,8$ & $96(59,3)$ & $51,3-66,9$ \\
\hline Pretas & $896(84,7)$ & $82,4-86,8$ & $753(84,0)$ & $81,5-86,4$ & $453(50,7)$ & $47,3-54,0$ \\
\hline \multicolumn{7}{|l|}{ Situação conjugal } \\
\hline Com companheiro & $901(82,7)$ & $80,3-84,9$ & $771(85,6)$ & $83,1-87,8$ & $474(52,7)$ & $49,4-56,0$ \\
\hline Sem companheiro & $189(17,3)$ & $15,1-19,7$ & $152(80,4)$ & $74,0-85,8$ & $93(49,2)$ & $41,9-56,6$ \\
\hline \multicolumn{7}{|l|}{ Número de gestações anteriores } \\
\hline$\geq 2$ & $234(21,5)$ & $75,9-80.9$ & $192(82,1)$ & $76,5-86,7$ & $134(57,3)$ & $47,3-54,1$ \\
\hline$<2$ & $854(78,5)$ & $19,1-24,1$ & $730(85,5)$ & $82,9-87,8$ & $432(50,7)$ & $47,3-54,1$ \\
\hline \multicolumn{7}{|l|}{ Início do pré-natal } \\
\hline Durante o 10 trimestre & $860(83,0)$ & $80,6-85,3$ & $752(87,4)$ & $85,0-89,6$ & $433(50,5)$ & $47,1-53,9$ \\
\hline Após o 10 trimestre & $176(17,0)$ & $14,7-19,4$ & $138(78,4)$ & $71,6-84,2$ & $107(60,8)$ & $53,2-68,1$ \\
\hline \multicolumn{7}{|l|}{ Consultas pré-natal } \\
\hline$\leq 3$ & $669(61,3)$ & $58,4-64,2$ & $523(78,2)$ & $74,9-81,3$ & $374(56,0)$ & $52,1-59,8$ \\
\hline$>3$ & $422(38,7)$ & $35,8-41,6$ & $401(95,9)$ & $92,5-96,9$ & $194(45,8)$ & $41,0-50,7$ \\
\hline \multicolumn{7}{|l|}{ Renda familiar (salários mínimos) } \\
\hline$>1$ & $796(76,5)$ & $73,8-79,0$ & $679(85,3)$ & $78,0-87,7$ & $429(52,8)$ & $49,3-56,3$ \\
\hline$\leq 1$ & $245(23,5)$ & $21,0-26,2$ & $204(83,3)$ & $82,6-87,7$ & $131(53,7)$ & $47,2-60,1$ \\
\hline \multicolumn{7}{|l|}{ Classe econômica } \\
\hline $\mathrm{A} / \mathrm{B}$ & $110(14,8)$ & $12,3-17,5$ & $92(83,6)$ & $75,4-90,0$ & $51(46,4)$ & $36,8-56,1$ \\
\hline C/D/E & $635(85,2)$ & $82,5-87,7$ & $511(80,5)$ & $77,2-83,5$ & $353(55,7)$ & $51,7-59,6$ \\
\hline \multicolumn{7}{|l|}{ História de aborto } \\
\hline Sim & $232(24,5)$ & $21,8-27,4$ & $196(84,5)$ & $79,2-88,9$ & $122(52,6)$ & $45,9-59,2$ \\
\hline Não & $714(75,5)$ & $72,6-78,2$ & $604(84,6)$ & $81,7-87,2$ & $383(53,7)$ & $50,0-57,4$ \\
\hline \multicolumn{7}{|l|}{ Fumante } \\
\hline Sim & $37(3,4)$ & $2,4-4,7$ & $33(89,2)$ & $74,6-97,0$ & $18(48,7)$ & $31,9-65,6$ \\
\hline Não & $1.040(96,6)$ & $95,3-97,6$ & $879(84,5)$ & $82,2-86,7$ & $542(52,2)$ & $49,1-55,3$ \\
\hline \multicolumn{7}{|l|}{ Ter algum problema de saúde ** } \\
\hline $\operatorname{Sim}$ & $542(50,3)$ & $47,3-53,4$ & $482(88,8)$ & $85,8-91,3$ & $286(52,9)$ & $48,6-57,1$ \\
\hline Não & $535(49,7)$ & $46,6-52,7$ & $432(80,8)$ & $77,1-84,0$ & $275(51,5)$ & $47,2-55,8$ \\
\hline
\end{tabular}

IC95\%: intervalo de 95\% de confiança.

* Gestantes que autorreferiram ser amarelas, brancas ou indígenas;

** Anemia, asma, tuberculose, pneumonia, diabetes, hipertensão, doença renal, infecção urinária e hemorragias.

Nota: existem perdas em algumas variáveis. Classe econômica é a variável com maior perda. 
Tabela 2

Prevalência de gestantes que utilizaram pelo menos um medicamento durante a gestação em cada um dos grupos farmacológicos da Anatomical Therapeutic Chemical Classification System (ATC) 21. Santo Antônio de Jesus, Bahia, Brasil, 2012-2014.

\begin{tabular}{|c|c|c|}
\hline \multirow[t]{2}{*}{ Classificação ATC } & \multicolumn{2}{|c|}{ Gestantes * } \\
\hline & $\begin{array}{c}\text { Durante a gestação } \\
\text { n (\%) }\end{array}$ & $\begin{array}{c}\text { Antes da gestação } \\
\text { n (\%) }\end{array}$ \\
\hline Sangue e órgãos hematopoiéticos - B & $779(71,4)$ & $20(1,8)$ \\
\hline Antianêmicos - B03 & $777(71,2)$ & $19(1,7)$ \\
\hline Vitamina B12 e ácido fólico - B03B & $686(62,9)$ & $15(1,4)$ \\
\hline Preparações contendo ferro - B03A & $566(51,9)$ & $5(0,5)$ \\
\hline Trato gastrointestinal e metabolismo - A & $292(26,8)$ & $90(8,2)$ \\
\hline Medicamentos para distúrbios gastrointestinais - A03 & $213(19,5)$ & $47(4,3)$ \\
\hline Belladonna e derivados - A03B & $99(9,1)$ & $36(3,3)$ \\
\hline Antiespasmódico em combinação com analgésicos - A03D & $77(7,1)$ & $7(0,6)$ \\
\hline Antiespasmódico e anticolinérgicos, associação - A03E & $41(3,8)$ & - \\
\hline Propulsivos - A03F & $13(1,2)$ & $4(0,4)$ \\
\hline Vitaminas - A11 & $85(7,8)$ & $23(1,1)$ \\
\hline Combinações de multivitaminas - A11A & $79(7,5)$ & $6(1,0)$ \\
\hline Medicamentos para distúrbios de acidez - A02 & $21(1,9)$ & $25(2,3)$ \\
\hline Antiácidos - A02A & $16(1,5)$ & $2(0,2)$ \\
\hline Medicamentos para úlcera péptica e refluxo gastroesofágico - A02B & $5(0,5)$ & $23(2,1)$ \\
\hline Sistema nervoso central - N & $239(21,9)$ & $199(18,2)$ \\
\hline Analgésicos - N02 & $238(21,8)$ & $187(17,1)$ \\
\hline Analgésicos não-opioides - N02B & $238(21,8)$ & $183(16,8)$ \\
\hline Sistema respiratório - $\mathrm{R}$ & $101(9,3)$ & $23(2,1)$ \\
\hline Anti-histamínicos sistêmicos - R06 & $97(8,9)$ & $15(1,4)$ \\
\hline Anti-histamínicos sistêmicos - R06A & $97(8,9)$ & $15(1,4)$ \\
\hline Sistema geniturinário e hormônios sexuais - G & $44(4,0)$ & $145(13,3)$ \\
\hline Hormônios sexuais e moduladores do sistema reprodutivo - G03 & $30(2,8)$ & $134(12,3)$ \\
\hline Hormônios contraceptivos de uso sistêmico - G03A & $2(0,2)$ & $115(10,5)$ \\
\hline Progestógenos - G03D & $28(2,6)$ & $2(0,2)$ \\
\hline Anti-infecciosos e antissépticos vaginais - G01 & $14(1,3)$ & $10(0,9)$ \\
\hline Anti-infecciosos e antissépticos, excluindo combinações com corticoides - G01A & $14(1,3)$ & $10(0,9)$ \\
\hline Anti-infecciosos de uso sistêmico - J & $36(3,3)$ & $37(3,4)$ \\
\hline Antibacterianos sistêmicos - J01 & $34(3,1)$ & $33(3,0)$ \\
\hline Antibacterianos beta-lactâmicos e penicilinas - J01C & $17(1,6)$ & $23(2,1)$ \\
\hline Outros antibacterianos beta-lactâmicos - J01D & $16(1,5)$ & $3(0,3)$ \\
\hline Outros antibacterianos - J01X & $5(0,5)$ & - \\
\hline Sistema cardiovascular - C & $26(2,4)$ & $23(2,1)$ \\
\hline Anti-hipertensivos - CO2 & $23(2,1)$ & $1(0,1)$ \\
\hline Agente antiadrenérgicos de ação central - C02A & $23(2,1)$ & $1(0,1)$ \\
\hline Medicamentos que agem no sistema renina-angiotensina - C09 & $1(0,1)$ & $10(1,2)$ \\
\hline Agentes beta-bloqueadores - C07 & $1(0,1)$ & $7(0,6)$ \\
\hline Sistema músculo esquelético - M & $24(2,2)$ & $153(14,0)$ \\
\hline Relaxantes musculares - M03 & $10(0,9)$ & $101(9,3)$ \\
\hline Relaxantes musculares de ação central - M03B & $10(0,9)$ & $101(9,3)$ \\
\hline Anti-inflamatórios e antirreumáticos - M01 & $14(1,3)$ & $63(5,8)$ \\
\hline Anti-inflamatórios e antirreumáticos não esteroides - M01A & $14(1,3)$ & $63(5,8)$ \\
\hline Outros & $11(1,0)$ & $18(1,6)$ \\
\hline Total & $1.091(167,9) * *$ & $1.091(124,8) * *$ \\
\hline
\end{tabular}

* Proporção de gestantes usuárias de medicamentos que fizeram uso daquele grupo;

** O total das porcentagens soma mais de 100,0\% porque várias gestantes utilizaram mais de um medicamento. 
Frequência de medicamentos utilizados antes e durante a gestação por grupo Anatomical Therapeutic Chemical Classification System (ATC) 21. Santo Antônio de Jesus, Bahia, Brasil, 2012- 2014.

\begin{tabular}{lcc}
\hline Classificação ATC & \multicolumn{2}{c}{ Gestantes * } \\
& Antes da gestação \\
& $\mathbf{n}(\%)$ \\
\hline Sangue e órgãos hematopoiéticos - B & $\mathbf{n}(\%)$ & $22(2,8)$ \\
Trato gastrointestinal e metabolismo - A & $1.278(59,7)$ & $97(12,5)$ \\
Sistema nervoso central - N & $361(16,9)$ & $231(19,7)$ \\
Sistema respiratório - R & $246(11,5)$ & $24(3,1)$ \\
Sistema geniturinário e hormônios sexuais - G & $108(5,1)$ & $153(19,7)$ \\
Anti-infecciosos de uso sistêmico - J & $44(2,1)$ & $37(4,8)$ \\
Sistema cardiovascular - C & $41(1,9)$ & $26(3,1)$ \\
Sistema musculoesquelético - M & $27(1,3)$ & $170(21,9)$ \\
Outros & $25(1,2)$ & $18(2,3)$ \\
Total & $11(0,2)$ & $778(100,0)$ \\
\hline
\end{tabular}

* Proporção de gestantes usuárias de medicamentos que fizeram uso daquele grupo.

As variáveis idade materna $\geq 30$ anos, cor de pele não preta, início do pré-natal após o 1o trimestre e as com três ou menos consultas pré-natal estão estatisticamente associadas à utilização de medicamentos antes da gestação; já as variáveis ter algum problema de saúde, início do pré-natal durante o 1o trimestre, mais de três consultas pré-natal e mais de nove anos de estudo estão estatisticamente associadas à utilização de medicamentos durante a gestação, ambos, na análise bivariada como descrito na Tabela 4.

Após a análise multivariada, encontrou-se que existe maior prevalência na utilização de medicamentos antes da gestação entre as gestantes com idade $\geq 30$ anos de idade, as não pretas, as que iniciaram o pré-natal depois do 1o trimestre e as que fazem parte da classe econômica C/D/E. Os fatores associados à utilização de medicamentos durante a gravidez que permaneceram no modelo após a análise multivariada foram escolaridade $\geq 11$ anos de estudo, ter feito três ou mais consultas pré-natal e ter algum problema de saúde (Tabela 4).

\section{Discussão}

Os resultados desse trabalho fazem parte de um estudo de coorte de gestantes acompanhadas no SUS, em uma cidade de pequeno porte do Nordeste do Brasil. Foram incluídas na pesquisa mulheres com companheiros, predominantemente pretas e com renda maior que um salário mínimo. A maior parte das gravidezes não foi planejada e mais de $80 \%$ das mulheres iniciaram o pré-natal no $1^{\circ}$ trimestre.

A prevalência do uso de medicamentos antes de diagnosticada a gravidez foi de $53 \%$, em sua maior parte por automedicação, sendo relaxante muscular como a associação de citrato de orfenadrina/ dipirona/cafeína, analgésicos como dipirona e paracetamol e contraceptivos orais combinados, os grupos de medicamentos mais utilizados. A comparação com as prevalências encontradas em países da Europa e nos Estados Unidos (14,2\% a 43\%) é dificultada pela variação no tempo de observação antes da gestação (30 dias a 3 anos) 6,7,8,9,10,23, pois, no presente estudo, não houve delimitação de um período recordatório específico no momento da coleta dos dados sobre o uso de medicamentos antes da gestação.

Com relação a outros estudos realizados no Brasil, o resultado foi ligeiramente superior ao verificado em Santa Catarina, onde aproximadamente 47\% das gestantes utilizaram medicamentos antes do diagnóstico gestacional 11 e inferior às prevalências de uso por mulheres na população geral verificadas em Brasília (59,8\%) e em Fortaleza, Estado do Ceará (55,6\%) 24,25. 


\section{Tabela 4}

Razões de prevalência bruta (RP) e ajustada (RPa) para uso de medicamentos antes e durante a gestação e características socioeconômicas e de saúde em gestantes. Santo Antônio de Jesus, Bahia, Brasil, 2012-2014 ( $N=1.091)$.

\begin{tabular}{|c|c|c|c|c|}
\hline \multirow[t]{2}{*}{ Características socioeconômicas e de saúde } & \multicolumn{2}{|c|}{ Antes da gestação } & \multicolumn{2}{|c|}{ Durante a gestação } \\
\hline & RP (IC95\%) & $\mathrm{RPa}(\mathrm{IC} 95 \%)$ & RP (IC95\%) & $\mathrm{RPa}(\mathrm{IC95 \% )}$ \\
\hline \multicolumn{5}{|l|}{ Idade materna (anos) } \\
\hline $18-24$ & 1,00 & 1,00 & 1,00 & - \\
\hline $25-29$ & $1,05(0,91-1,21)$ & - & $1,01(0,95-1,07)$ & - \\
\hline $30-45$ & $1,14(1,00-1,30)$ & $1,38(1,08-1,78)$ * & $1,02(0,96-1,08)$ & - \\
\hline \multicolumn{5}{|l|}{ Escolaridade (anos) } \\
\hline$\leq 8$ & 1,00 & - & 1,00 & 1,00 \\
\hline $9-11$ & $1,04(0,92-1,17)$ & - & $1,05(1,00-1.11)$ & - \\
\hline$>11$ & $1,23(0,99-1,52)$ & - & $1,11(1,02-1,21)$ & $1,10(1,01-1,18)$ * \\
\hline \multicolumn{5}{|l|}{ Cor de pele } \\
\hline Não pretas & $1,17(1,02-1,35)$ & $1,21(1,04-1,44)$ * & 1,00 & - \\
\hline Pretas & 1,00 & 1,00 & $0,96(0,90-1,03)$ & - \\
\hline \multicolumn{5}{|l|}{ Situação conjugal } \\
\hline Sem companheiro & 1,00 & - & 1 & - \\
\hline Com companheiro & $1,07(0,91-1,25)$ & - & $1,06(0,97-1,14)$ & - \\
\hline \multicolumn{5}{|l|}{ Número de gestações } \\
\hline$<2$ & 1,00 & 1,00 & 1,00 & - \\
\hline$\geq 2$ & $1,12(0,99-1,28)$ & - & $0,95(0,89-1,02)$ & - \\
\hline \multicolumn{5}{|l|}{ Início do pré-natal } \\
\hline Após o 1o trimestre & $1,20(1,05-1,38)$ & $1,17(1,01-1,36)$ * & 1,00 & - \\
\hline Durante o 1 o trimestre & 1,00 & 1,00 & $1,11(1,02-1,20)$ & - \\
\hline \multicolumn{5}{|l|}{ Consultas de pré-natal } \\
\hline$\leq 3$ & 1,00 & & 1,00 & 1,00 \\
\hline$>3$ & $0,81(0,72-0,92)$ & - & $1,21(1,16-1,27)$ & $1,20(1,15-1,26)$ * \\
\hline \multicolumn{5}{|l|}{ Renda familiar (salários mínimos) } \\
\hline$\leq 1$ & 1,00 & - & 1,00 & - \\
\hline$>1$ & $0,98(0,86-1,12)$ & - & $1,02(0,96-1,09)$ & - \\
\hline \multicolumn{5}{|l|}{ Classe econômica } \\
\hline C/D/E & $1,20(0,97-1,49)$ & $1,28(1,01-1,61)$ * & 1,00 & - \\
\hline $\mathrm{A} / \mathrm{B}$ & 1,00 & 1,00 & $1,03(0,94-1,13)$ & - \\
\hline \multicolumn{5}{|l|}{ História de aborto } \\
\hline Não & 1,00 & - & 1,00 & - \\
\hline Sim & $0,97(0,85-1,12)$ & - & $0,99(0,93-1,06)$ & - \\
\hline \multicolumn{5}{|l|}{ Fumante } \\
\hline Não & 1,00 & - & 1,00 & - \\
\hline $\operatorname{Sim}$ & $0,93(0,66-1,30)$ & - & $1,05(0,94-1,18)$ & - \\
\hline \multicolumn{5}{|l|}{ Ter algum problema de saúde ** } \\
\hline Não & 1,00 & - & 1,00 & 1,00 \\
\hline Sim & $1,02(0,91-1,15)$ & - & $1,09(1,04-1,15)$ & $1,07(1,02-1,13)$ * \\
\hline
\end{tabular}

IC95\%: intervalo de 95\% de confiança.

* Variáveis que se mantiveram no modelo final da regressão de Poisson. As caselas não preenchidas correspondem as variáveis que não permaneceram nesse modelo;

** Gestante ter autorreferido anemia, asma, tuberculose, pneumonia, diabetes, hipertensão, doença renal, infecção urinária e hemorragias. 
A dipirona foi o princípio ativo, isolado ou em associação, mais utilizado pelas mulheres antes da gestação $(19,1 \%)$, dado que difere do encontrado por Crespin et al. ${ }^{26}$ na França e por outro estudo brasileiro 11 no qual o analgésico mais utilizado foi o paracetamol. Continuam existindo dúvidas sobre a segurança da dipirona para o concepto, embora novas evidências positivas tenham surgido 27,28,29,30.

Os contraceptivos orais foram a segunda classe de medicamentos mais utilizados antes da gestação, dados que sugerem o incorreto uso deles e falhas no acesso às informações necessárias ao uso adequado. Mulheres que não têm conhecimento de sua gravidez podem continuar a usar esses medicamentos após a concepção, expondo o embrião a riscos evitáveis 30 .

Investigação retrospectiva realizada no País de Gales, que incluiu 37 casos de defeitos do tubo neural, constatou um aumento de 2,5 vezes no risco desse problema em bebês expostos a contraceptivos orais no início da gestação 31 . Estudo húngaro de caso-controle, abrangendo 537 casos, observou risco aumentado de defeitos nos membros de $1,7 \mathrm{vez} 32$. No entanto, estudo caso-controle multicêntrico realizado nos Estados Unidos concluiu que mulheres que usam contraceptivos orais durante o início da gravidez não têm risco aumentado para a maioria dos tipos de más-formações congênitas maiores 33. Em uma meta-análise, envolvendo 12 estudos prospectivos, Bracken 34 também concluiu que os contraceptivos orais não foram associados com risco aumentado de defeitos cardíacos congênitos e defeitos de redução de membros. De qualquer forma, considera-se necessário orientar corretamente o uso de contraceptivos orais na população feminina em idade fértil, tanto para um planejamento familiar adequado quanto para a diminuição dos riscos para o feto.

Após o ajuste para situação socioeconômica e de saúde, foram encontrados os seguintes fatores associados à utilização de medicamentos antes da gestação: gestantes com idade $\geq 30$ anos, não pretas, que iniciaram o pré-natal depois do 1o trimestre e que fazem parte da classe econômica C/D/E. A carência de estudos semelhantes dificulta a comparação dos resultados. Na Sérvia, Odalovic et al. 12, em análise bivariada, encontraram que gestantes com mais de 30 anos têm uma chance aumentada de utilizar medicamentos antes da gestação (odds ratio - OR = 3,03; IC95\%: 1,16-8,00), mas essa associação não se manteve na análise multivariada, diferente do encontrado no presente trabalho em que a idade materna se manteve no modelo final. Uma justificativa para essa diferença se deve ao fato de que na população geral as mulheres brasileiras com mais de 30 anos já têm chance maior de utilizar medicamentos 35 , o que se manteria durante o período periconcepcional.

Mulheres que iniciaram o pré-natal no 1o trimestre da gestação tiveram uma menor prevalência na utilização de medicamentos antes da gravidez, sugerindo um maior cuidado com a própria saúde ou que já estivessem planejando engravidar e, por isso, evitando a automedicação.

No presente estudo, encontrou-se uma frequência no uso de pelo menos um medicamento durante a gravidez em $84,7 \%$ das gestantes, prevalência semelhante à encontrada em trabalhos no Brasil e em outros países. Estudo realizado nos Estados Unidos mostrou que, em 2008, 90\% das mulheres pesquisadas utilizaram pelo menos um medicamento durante a gravidez 36; na Hungria, de acordo com Czeizel $37,70 \%$ das gestantes foram tratadas com algum medicamento em qualquer período da gestação; trabalhos realizados no Brasil mostraram variação entre 82,9 e 97,4\% no uso de pelo menos um medicamento durante a gestação, incluindo vitaminas e suplementos 17,18. É evidente a grande contribuição de medicamentos recomendados em protocolos pela OMS e pelo Ministério da Saúde, no caso do Brasil, tais como ácido fólico, sulfato ferroso e algumas vitaminas 38 .

É observado que existem variações de prevalência entre os locais de estudo; essas diferenças seriam reflexo das características dos serviços de atenção à saúde, dos problemas de saúde e das diferenças culturais entre os locais estudados 39 , o que poderia explicar as diferenças de perfis também entre cidades brasileiras. Contudo, sugere-se também que o uso de medicamentos seria não apenas reflexo, mas faria parte dessas diferenças 19 .

Mais da metade utilizada dos medicamentos foi com prescrição, e a maior parte iniciada no $1^{\circ}$ trimestre, o que reflete o acompanhamento pré-natal. Mesmo levando em conta a larga contribuição dos medicamentos recomendados em protocolos do Ministério da Saúde para uso na gestação como os antianêmicos, ácido fólico e vitaminas 38 , torna-se evidente a larga prática da prescrição médica como medida de intervenção na gravidez.

Exemplo disso é o uso generalizado de antianêmicos $(71,2 \%)$, já que ainda se questiona até que ponto a suplementação profilática de ferro seria necessária e, uma vez diagnosticada a anemia, quais 
os esquemas de tratamento mais eficientes, visto que os diferentes grupos biológicos podem responder de diversas maneiras 40,41.

Os analgésicos foram utilizados por 21,9\% das gestantes, sendo o paracetamol o mais prevalente (com 21,3\%), isolado ou em associações, seguindo-se a dipirona com 7,7\%. Particularmente, o paracetamol é considerado de uso seguro na gestação, e as dúvidas quanto à dipirona já foram comentadas 42 .

Entre os medicamentos que atuam no aparelho digestivo, a escopolamina isolada e associada a analgésicos (18,9\%), foi o princípio ativo mais utilizado; em estudo realizado em gestantes na cidade de São Paulo, essa prevalência foi de 46,8\% 43 e, em Campinas (Estado de São Paulo), a escopolamina correspondeu a 70,2\% dos medicamentos utilizados por grávidas 11,16,44. O uso desse fármaco está relacionado à sua indicação no tratamento de cólicas no início da gestação, sugestivo de ameaça de abortamento ou mais provavelmente de uso inadvertido em associações com analgésico, expondo a gestante a riscos pouco conhecidos.

No presente trabalho, dentre os fatores associados à utilização de medicamentos durante a gestação, ter 11 anos ou mais de estudo teve efeito positivo. Em investigação realizada por Mengue et al. 17, em Porto Alegre, Estado do Rio Grande do Sul, foi encontrada associação semelhante, com aumento na chance do consumo de medicamentos em gestantes com maior escolaridade (OR = 1,65; IC95\%: 1,30-2,10); os autores sugerem que uma maior escolaridade indica um melhor nível socioeconômico, facilitando o acesso ao serviço de saúde e ao medicamento.

O número de consultas pré-natal também se mostrou associado ao uso de medicamentos durante a gravidez, associação já verificada entre gestantes das cidades de Campinas 16 e de Rio Branco (Estado do Acre) ${ }^{18}$. Na última, ter feito mais de seis consultas de pré-natal aumentou em 1,78 vez (IC95\%: 1,33-2,38) a chance do uso de medicamentos como antianêmicos e vitaminas 18 .

Neste trabalho, ter algum problema de saúde apresentou associação positiva e significante com o uso de medicamentos durante a gravidez na análise bruta e na análise ajustada. Diversos estudos mostram que o consumo de medicamentos está associado a ter problemas de saúde, inclusive durante a gestação 18,45,46. Mesmo em países desenvolvidos, como o Canadá, por exemplo, um estudo de base populacional encontrou que ter uma doença crônica (OR = 2,01; IC95\%: 1,98-2,05) deixou as gestantes mais susceptíveis a receberem prescrições de medicamentos durante a gravidez 46 . Esses fatores aumentaram o contato entre o prescritor e a gestante, e isso foi associado a um maior consumo de medicamentos, que pode ter ocorrido por real necessidade de tratamento para uma patologia ou por causa de queixas comuns da gravidez, sendo elas muitas vezes condições autolimitadas que poderiam ser resolvidas sem intervenções farmacoterapêuticas. Estudos sobre a função social da prescrição de medicamentos mostram que para o médico a prescrição não é apenas a documentação de sua intervenção, mas um sinal de atenção com o paciente. Do ponto de vista do paciente, a prescrição de medicamentos feita pelo médico é esperada, pois comprovaria que suas queixas estão sendo valorizadas 47 .

As possíveis limitações do presente estudo dizem respeito ao viés recordatório, sobre a utilização de medicamentos antes e durante a gestação, fato que pode gerar uma subestimação nas prevalências dos desfechos. Para minimizar o problema, procedimentos tais como o uso de questionário padronizado e testado, equipe bem treinada, padronização da coleta de dados e validação dos dados por comparação das informações obtidas pelos questionários com aquelas registradas nos cartões de pré-natal foram adotados.

No caso do desfecho, uso de medicamentos anteriormente à gravidez, faltou especificar um período recordatório que indicasse quanto tempo antes de engravidar estava se captando a informação; a gestante pode ter incluído informações sobre uso de medicamentos no início da gestação e não antes do diagnóstico gestacional.

O estudo foi realizado somente na zona urbana da cidade por conta das dificuldades enfrentadas para a coleta na zona rural e captou apenas as gestantes que frequentavam as Unidades Básicas de Saúde; assim, o trabalho não representa a totalidade das gestantes da cidade.

O estudo põe em evidência a lógica da intervenção farmacológica na vida das pessoas desde a gestação, apesar da carência de informações seguras que fundamentem o uso de medicamentos nessa fase da existência. Verificou-se uma elevada prevalência do uso de medicamentos por mulheres antes e, sobretudo, durante a gestação, por automedicação e por prescrição médica, sugerindo que a exposição do concepto a fármacos e o acompanhamento pré-natal fazem parte de uma mesma lógica intervencionista. 
O uso de medicamentos durante a gestação aparece associado com fatores relativos ao acompanhamento pré-natal tais como o período de início do acompanhamento e a presença de problemas de saúde, o que aponta para a necessidade de intervenções que previnam os agravos durante o período gestacional.

Diante desses dados e mesmo considerando as limitações apontadas pelo estudo, sugere-se um acompanhamento pré-natal com a presença de um farmacêutico juntamente com a equipe, e ele deve ser menos centrado em intervenções farmacológicas e mais em orientação e educação que possam ajudar a gestante inclusive a compreender os riscos e cuidados inerentes ao uso de medicamentos, aprendizado que trará benefícios a ela e a sua família.

\section{Colaboradores}

D. B. Costa participou da concepção do estudo, processamento, análise e interpretação dos dados, revisão da literatura e redação do artigo. H. L. L. Coelho contribuiu com a concepção do estudo e revisão final do artigo. D. B. Santos colaborou na concepção do estudo, processamento, organização, análise e interpretação dos dados e revisão final do artigo.

\section{Agradecimentos}

Ao CNPq (Processo 481509/2012-7. Edital Universal - 14/2012) e à FAPESB (PPP0073/2011 Edital $025 / 2010$ ) pelo apoio financeiro ao trabalho de campo e financiamento do projeto de pesquisa. Às gestantes que compõem o estudo de coorte do Núcleo de Investigação em Saúde Materno-Infantil (NISAMI) do Centro de Ciências da Saúde, Universidade Federal do Recôncavo da Bahia, Brasil. 


\section{Referências}

1. Matthews SJ, McCoy C. Thalidomide: a review of approved and investigational uses. Clin Ther 2003; 25:342-95.

2. Berglund F, Flodh H, Lundborg P, Prame B, Sannerstedt R. Drug use during pregnancy and breastfeeding: a classification system for drug information. Acta Obstet Gynecol Scand Suppl 1984; 63(S126):1-55.

3. Forfar JO, Nelson MM. Epidemiology of drugs taken by pregnant women: drugs that may affect the fetus adversely. Clin Pharmacol Ther 1973; 14:632-42.

4. Gomes KR, Moron AF, Silva RS, Siqueira AAF. Prevalence of use of medicines during pregnancy and its relationship to maternal factors. Rev Saúde Pública 1999; 33:246-54.

5. Thorpe PG, Gilboa SM, Hernandez-Diaz S, Lind J, Cragan JD, Briggs G, et al. Medications in the first trimester of pregnancy: most common exposures and critical gaps in understanding fetal risk. Pharmacoepidemiol Drug Saf 2013; 22:1013-8.

6. Engeland A, Bramness JG, Daltveit AK, Rønning M, Skurtveit S, Furu K. Prescription drug use among fathers and mothers before and during pregnancy. A population-based cohort study of 106,000 pregnancies in Norway 2004-2006. Br J Clin Pharmacol 2008; 65:653-60.

7. Bakker MK, Jentink J, Vroom F, Van Den Berg PB, De Walle HE, De Jong-Van Den Berg LT. Drug prescription patterns before, during and after pregnancy for chronic, occasional and pregnancy-related drugs in the Netherlands. BJOG 2006; 113:559-68.

8. Stephansson O, Granath F, Svensson T, Haglund B, Ekbom A, Kieler H. Drug use during pregnancy in Sweden - assessed by the Prescribed Drug Register and the Medical Birth Register. Clin Epidemiol 2011; 3:43-50.

9. Hardy JR, Leaderer BP, Holford TR, Hall GC, Bracken MB. Safety of medications prescribed before and during early pregnancy in a cohort of 81,975 mothers from the UK General Practice Research Database. Pharmacoepidemiol Drug Saf 2006; 15:555-64.

10. Odalovic M, Kovacevic SV, Ilic K, Sabo A, Tasic L. Drug use before and during pregnancy in Serbia. Int J Clin Pharm 2012; 34:719-27.

11. Lunardi-Maia T, Schuelter-Trevisol F, Galato D. Uso de medicamentos no primeiro trimestre de gravidez: avaliação da segurança dos medicamentos e uso de ácido fólico e sulfato ferroso. Rev Bras Ginecol Obstet 2014; 36:541-7.

12. Odalovic M, Kovacevic SV, Nordeng H, Ilic K, Sabo A, Tasic L. Predictors of the use of medications before and during pregnancy. Int J Clin Pharm 2013; 35:408-16.
13. Bánhidy F, Lowry RB, Czeizel AE. Risk and benefit of drug use during pregnancy. Int J Med Sci 2005; 2:100-6.

14. Lacroix I, Damase-Michel C, Lapeyre-Mestre M, Montastruc JL. Prescription of drugs during pregnancy in France. Lancet 2000; 356:1735-6.

15. Lupattelli A, Spigset O, Twigg MJ, Zagorodnikova K, Mårdby A-C, Moretti ME, et al. Medication use in pregnancy: a cross-sectional, multinational web-based study. BMJ Open 2014; 4:e004365.

16. Fonseca MRCC, Fonseca E, Bergsten-Mendes G. Prevalência do uso de medicamentos na gravidez: uma abordagem farmacoepidemiológica. Rev Saúde Pública 2002; 36:205-12.

17. Mengue SS, Schenkel EP, Schmidt MI, Duncan BB. Fatores associados ao uso de medicamentos durante a gestação em seis cidades brasileiras. Cad Saúde Pública 2004; 20:1602-8.

18. Andrade AM, Ramalho AA, Koifman RJ, Dotto LMG, Cunha MA, Opitz SP. Fatores associados ao uso de medicamentos na gestação em primigestas no Município de Rio Branco, Acre, Brasil. Cad Saúde Pública 2014; 30:1042-56.

19. Mengue SS, Schenkel EP, Duncan BB, Schmidt MI. Uso de medicamentos por gestantes em seis cidades brasileiras. Rev Saúde Pública 2001; 35:41520.

20. Olesen C, Thrane N, Henriksen TB, Ehrenstein V, Olsen J. Associations between socio-economic factors and the use of prescription medication during pregnancy. Eur J Clin Pharmacol 2006; 62:547-53.

21. WHO International Working Group for Drug Statistics Methodology; WHO Collaborating Centre for Drug Statistics Methodology; WHO Collaborating Centre for Drug Utilization Research and Clinical Pharmacological Services. Introduction to drug utilization research. Geneva: World Health Organization; 2003.

22. Barros AJ, Hirakata VN. Alternatives for logistic regression in cross-sectional studies: an empirical comparison of models that directly estimate the prevalence ratio. BMC Med Res Methodol 2003; $3: 21$.

23. Roberson EK, Hurwitz EL. Prescription drug use during and immediately before pregnancy in Hawai'i: findings from the Hawai'i Pregnancy Risk Assessment Monitoring System, 2009-2011. Hawaii J Med Public Health 2014; 73:382-6.

24. Galvão TF, Silva MT, Gross R, Pereira MG. Medication use in adults living in Brasilia, Brazil: a cross-sectional, population-based study. Pharmacoepidemiol Drug Saf 2014; 23:507-14.

25. Arrais PSD, Brito LL, Barreto ML, Coelho HLL. Prevalência e fatores determinantes do consumo de medicamentos no Município de Fortaleza, Ceará, Brasil. Cad Saúde Pública 2005; 21:1737-46. 
26. Crespin S, Bourrel R, Hurault-Delarue C, Lapeyre-Mestre M, Montastruc JL, Damase-Michel C. Drug prescribing before and during pregnancy in south west France: a retrolective study. Drug Saf 2011; 34:595-604.

27. Espiridião S, Oliveira-Filho R, Doine A, Simões M, Focchi G, Evêncio Neto J, et al. Morphological and biochemical action of dipyrone on rat placenta. Gen Pharmacol 1996; 27:505-7.

28. Bar-Oz B, Clementi M, Di Giantonio E, Greenberg R, Beer M, Merlob P, et al. Metamizol (dipyrone, optalgin) in pregnancy, is it safe? A prospective comparative study. Eur J Obstet Gynecol Reprod Biol 2005; 119:176-9.

29. Bánhidy F, Ács N, Puhó E, Czeizel AE. A population-based case-control teratologic study of oral dipyrone treatment during pregnancy. Drug Saf 2007; 30:59-70

30. Chen X-K, Wen SW, Sun L-M, Yang Q, Walker MC, Krewski D. Recent oral contraceptive use and adverse birth outcomes. Eur J Obstet Gynecol Reprod Biol 2009; 144:40-3.

31. Kasan PN, Andrews J. Oral contraception and congenital abnormalities. BJOG 1980; 87:545-51.

32. Czeizel AE, Kodaj I. A changing pattern in the association of oral contraceptives and the different groups of congenital limb deficiencies. Contraception 1995; 51:19-24.

33. Waller DK, Gallaway MS, Taylor LG, Ramadhani TA, Canfield MA, Scheuerle A, et al. Use of oral contraceptives in pregnancy and major structural birth defects in offspring. Epidemiology 2010; 21:232-9.

34. Bracken MB. Oral contraception and congenital malformations in offspring: a review and meta analysis of the prospective studies. Obstet Gynecol 1990; 76(3 Pt 2):552-7.

35. Bertoldi AD, Barros A, Hallal PC, Lima RC. Utilização de medicamentos em adultos: prevalência e determinantes individuais. Rev Saúde Pública 2004; 38:228-38.

36. Mitchell AA, Gilboa SM, Werler MM, Kelley KE, Louik C, Hernández-Díaz S, et al. Medication use during pregnancy, with particular focus on prescription drugs: 1976-2008. Am J Obstet Gynecol 2011; 205:51.e.1-e.8.

37. Czeizel A. Drug exposure in pregnant women. Lupus 2004; 13:740-5.
38. Departamento de Ações Programáticas Estratégicas, Secretaria de Atenção à Saúde, Ministério da Saúde. Pré-natal e puerpério: atenção qualificada e humanizada - manual técnico. Brasília: Ministério da Saúde; 2005.

39. Medication during pregnancy: an intercontinental cooperative study. Collaborative Group on Drug Use in Pregnancy (C.G.D.U.P.). Int J Gynaecol Obstet 1992; 39:185-96.

40. Szajewska H, Ruszczynski M, Chmielewska A. Effects of iron supplementation in nonanemic pregnant women, infants, and young children on the mental performance and psychomotor development of children: a systematic review of randomized controlled trials. Am J Clin Nutr 2010; 91:1684-90.

41. Peña-Rosas JP, De-Regil LM, Dowswell T, Viteri FE. Intermittent oral iron supplementation during pregnancy. Cochrane Database Syst Rev 2012; (7):CD009997.

42. Sharpe CR, Franco EL. Use of dipyrone during pregnancy and risk of Wilms' tumor. Brazilian Wilms' Tumor Study Group. Epidemiology 1996; 7:533-5.

43. Maeda ST, Secoli SR. Use and cost of medication in low risk pregnant women. Rev Latinoam Enferm 2008; 16:266-71.

44. Oliveira Filho AD, Gama DP, Leopardi MG, Dias JMG, Lyra Júnior DP, Neves SJF. Aderência autorreferida a medicamentos prescritos durante a gestação. Rev Bras Ginecol Obstet 2012; 34:147-52.

45. Donati S, Baglio G, Spinelli A, Grandolfo M. Drug use in pregnancy among Italian women. Eur J Clin Pharmacol 2000; 56:323-8.

46. Smolina K, Hanley G, Mintzes B, Oberlander T, Morgan S. Trends and determinants of prescription drug use during pregnancy and postpartum in British Columbia, 2002-2011: a population-based cohort study. PloS One 2014; 10:e0128312-e.

47. Pepe VLE, Osório-de-Castro CGS. A interação entre prescritores, dispensadores e pacientes: informação compartilhada como possível benefício terapêutico. Cad Saúde Pública 2000; 16:815-22. 


\section{Abstract}

This cross-sectional prospective nested cohort study aimed to assess the prevalence of use of medication before and during pregnancy and associated factors in women in a municipality in the countryside of Bahia State, Brazil. Data were collected with a structured questionnaire applied to pregnant women at their prenatal visits at health units. Prevalence rates for use of medication before and during pregnancy were $52.1 \%$ and $84.7 \%$, respectively. The following were associated with use of medication before pregnancy: age $\geq 30$ years, non-white skin color, first prenatal visit after the 1 st trimester, and economic classes $C / D / E$. There was an increase in medication during pregnancy among women with $\geq 11$ years of schooling, women with more than three prenatal visits, and those with some health problem. Pregnant women are exposed to medication before and during pregnancy, notwithstanding the lack of secure information to back the use of medicines during this phase; such use is associated with factors pertaining to prenatal follow-up, suggesting the need for more active participation by pharmacists in orientation and support for rational use of medicines.

Drug Utilization; Pregnancy;

Pharmacoepidemiology

\section{Resumen}

El Estudio transversal situado en una cohorte prospectiva, con el objetivo de evaluar la prevalencia y factores asociados a la utilización de medicamentos en gestantes antes y durante el embarazo en un municipio del interior de la región de Bahía, Brasil. La información fue recogida mediante un cuestionario estructurado, aplicado a las embarazadas en el momento del seguimiento prenatal en unidades de salud del municipio. La prevalencia para el consumo de medicamentos antes y durante la gestación fue un 52,1\% y un 84,7\%, respectivamente. Tras el análisis, los siguientes factores estaban asociados a la utilización de medicamentos antes de la gestación: $\geq 30$ años de edad; no afro-brasileñas; quienes comenzaron con el seguimiento prenatal tras el $1^{\circ}$ trimestre, $y$ las que forman parte de la clase económica $\mathrm{C} / \mathrm{D} / \mathrm{E}$. Existe un aumento de prevalencia de utilización de medicamentos durante la gestación entre las gestantes con una escolaridad $\geq 11$ años de estudio, haber realizado más de tres consultas prenatales y que tengan algún problema de salud. Las gestantes están expuestas al uso de medicamentos antes y durante la gestación, a pesar de la carencia de información segura que fundamente el uso de medicamentos en esa fase, y ese uso está asociado a factores relativos al seguimiento prenatal, sugiriéndose la inclusión más activa del farmacéutico en el equipo para la orientación y apoyo al uso racional de medicamentos.

Utilización de Medicamentos; Embarazo;

Farmacoepidemiología
Recebido em 04/Ago/2015

Versão final reapresentada em 03/Mar/2016

Aprovado em 18/Abr/2016 